\title{
Acurácia do Desempenho Funcional em Idosos Saudáveis, com Comprometimento Cognitivo Leve e Doença de Alzheimer
}

\author{
Natalia Lemos Fransen ${ }^{1}$ \\ Orcid.org/0000-0001-6823-6317 \\ Maila Holz ${ }^{1}$ \\ Orcid.org/0000-0001-8648-4234 \\ Andressa Pereira ${ }^{2}$ \\ Orcid.org/0000-0002-2110-2095 \\ Rochele Paz Fonseca ${ }^{1}$ \\ Orcid.org/0000-0001-6767-8439 \\ Renata Kochhann ${ }^{*}, 1$ \\ Orcid.org/0000-0002-6328-8131
}

${ }^{1}$ Pontificia Universidade Católica do Rio Grande do Sul, Porto Alegre, RS, Brasil

${ }^{2}$ Universidade do Vale do Rio dos Sinos, São Leopoldo, RS, Brasil

\section{Resumo}

Estabelecer o continuum de declínio cognitivo e funcional no envelhecimento normal para o patológico é desafiador. Nesta transição está o Comprometimento Cognitivo Leve (CCL), definido por prejuízo sutil na cognição e funcionalidade. Quando o prejuízo funcional aumenta preenche critérios para demência. Assim, determinar a acurácia diagnóstica da avaliação funcional é imprescindível. Com isso o objetivo foi comparar o desempenho funcional através da avaliação direta versus indireta e avaliar a acurácia do desempenho destas avaliações entre controles, pacientes com CCL e com doença de Alzheimer (DA). Participaram 90 indivíduos ( $n=27$ controles, $n=35$ pacientes com CCL e $n=28$ com DA). Foram utilizadas a Activities of Daily Living Questionnaire (ADL-Q) e a Direct Assessment of Functional Status (DAFS-R). Foram realizadas MANCOVA para comparar o desempenho funcional direto entre os participantes e curvas ROC para verificar a acurácia diagnóstica das escalas entre os grupos. $\mathrm{O}$ desempenho da avaliação direta demonstrou diferenciar controles de CCL e CCL de DA. Ambas as escalas mostraram diferenciar CCL e DA, e a DAFS-R controles de CCL. Assim, a DAFS-R evidenciou melhor acurácia do desempenho funcional nestes grupos.

Palavras-chave: Desempenho funcional, envelhecimento, Comprometimento Cognitivo Leve, doença de Alzheimer.

* Endereço para correspondência: Av. Ipiranga, 6681, prédio 11 - $9^{\circ}$ andar, sala 932, Porto Alegre, RS, Brasil 90619-900. Fone: (51) 33207742. E-mail: renata.kochhann@gmail.com

Agradecimentos: Ao conselho Nacional de Desenvolvimento Científico e Tecnológico (CNPq), Coordenação de Aperfeiçoamento de Pessoal de Nível Superior (Capes), Fundação de Amparo à Pesquisa do Estado do Rio Grande do Sul (FAPERGS). 


\title{
Accuracy of Functional Performance in Healthy Elderly Subjects, with Mild Cognitive Impairment and Alzheimer's Disease
}

\begin{abstract}
It is challenging to establish the continuum of cognitive and functional decline in normal to pathological aging. In this transition is the Mild Cognitive Impairment (MCI), defined by subtle impairment in cognition and functionality. When functional impairment increases, it meets the criteria for dementia. Thus, it is essential to determine an accurate diagnosis from the functional evaluation. The aim of this study was to compare functional performance through direct versus indirect assessments, verify the relationship between them, and evaluate the accuracy of it within the control group, MCI and Alzheimer's disease (AD) patients. Ninety subjects ( $n=27$ controls, $n=35 \mathrm{MCI}$ and $n=28 \mathrm{AD}$ ) participated in the study. We used the Activities of the Daily Living Questionnaire (ADL-Q) and the Direct Assessment of Functional Status (DAFS-R). MANCOVA was performed to compare the direct functional performance, and ROC curves were used to verify the diagnostic accuracy of the scales among groups. The performance of the direct assessment showed differences between the controls and MCI, and between MCI and AD subjects. Both scales showed differences between MCI and AD, and DAFS-R showed differences between controls and MCI. Thus, DAFS-R demonstrated better accuracy of functional performance in these groups.
\end{abstract}

Keywords: Functional performance, Aging, Mild Cognitive Impairment, Alzheimer's disease.

\section{Precisión del Rendimiento Funcional en Personas Mayores Sanas, con Deterioro Cognitivo Leve y Enfermedad de Alzheimer}

\section{Resumen}

Establecer el deterioro cognitivo y funcional en el envejecimiento normal y de la enfermedad es desafiador. Esta transición es el Deterioro Cognitivo Leve (DCL), definido por deterioro sutil en la cognición y la funcionalidad. Cuando aumenta el deterioro funcional cumple con los criterios para la demencia. Por lo tanto, para determinar la exactitud diagnóstica de evaluación funcional es esencial. Por lo tanto, el objetivo fue comparar el rendimiento funcional a través de directa frente a la evaluación indirecta, verificar la relación de estas actuaciones, evaluar la exactitud de los resultados de estas evaluaciones entre los controles, los pacientes con DCL y enfermedad de Alzheimer (EA). Participaron 90 sujetos ( $n=27$ controles, $n=35$ pacientes DCL y $n=28$ EA). Se utilizaron Activities of Daily Living Questionnaire (ADL-Q) y Direct Assessment of Functional Status (DAFS-R). Se realizaron MANCOVA para comparar el rendimiento funcional directo entre los participantes y las curvas ROC para verificar la precisión diagnóstica de las escalas entre los grupos. El rendimiento de la evaluación directa mostró diferenciar los controles de DCL, y DCL de EA. Ambas escalas mostraron diferenciar DCL y EA, y DAFS-R controles de DCL. Por lo tanto, DAFS-R mostró una mejor precisión del rendimiento funcional en estos grupos.

Palabras clave: Rendimiento funcional, envejecimiento, Deterioro Cognitivo Leve, enfermedad de Alzheimer.

A demência ocasionada pela doença de Alzheimer (DA) tem início insidioso podendo levar meses, ou anos para a piora cognitiva e funcional (Albert et al., 2011; McKhann et al., 2011). A distinção entre o envelhecimento nor- mal e patológico é um processo de difícil definição, uma vez que se caracteriza-se como um possível continuum. Nessa evolução, destaca-se o Comprometimento Cognitivo Leve (CCL) em que já se encontram prejuízos na cognição, mas 
com sutil impacto na vida diária, acometendo atividades mais complexas do indivíduo sem prejudicar seu nível de independência (Albert et al., 2011). Em vista desta difícil definição entre o envelhecimento saudável e patológico, a acurácia diagnóstica da avaliação funcional direta versus indireta é imprescindível.

Em geral, o início dos sintomas da DA e do CCL é apresentado por déficits cognitivos com apresentação amnéstica, ou não-amnéstica (Petersen et al., 2009). Nos quadros não-amnésticos os déficits podem ser de linguagem, atenção, percepção, agnosia, cognição social e/ou funções executivas, como, alteração de raciocínio, planejamento, flexibilidade cognitiva, controle inibitório, memória de trabalho, julgamento e resolução de problemas (American Psychiatric Association [APA], 2014; Frota et al., 2011; McKhann et al., 2011). Devido ao prejuízo cognitivo, o paciente pode apresentar, desde o início, queixas para executar tarefas que, anteriormente, eram habituais, como, preparar refeições, pagar contas e fazer compras (Frota et al., 2011).

Um estudo demonstrou uma taxa de 6,7 vezes maior de pessoas com CCL desenvolverem DA (Boyle, Wilson, Aggarwal, Tang, \& Bennett, 2006) do que indivíduos idosos saudáveis. Idosos com CCL apresentaram diminuição do funcionamento cognitivo mais rapidamente a cada ano do que pessoas com envelhecimento normal (Boyle et al., 2006). Apesar dos critérios diagnósticos de CCL evidenciarem preservação funcional, estudos sugerem que pacientes com CCL podem já ter prejuízos nas atividades de vida diárias (AVD) mais complexas, como lidar com as finanças e habilidades relacionadas a compras (Pereira, Oliveira, Diniz, Forlenza, \& Yassuda, 2010). Embora na DA o comprometimento das AVD é maior do que em pacientes com CCL (Marshall et al., 2011), informantes podem observar prejuízo no funcionamento diário de idosos com CCL quando comparado ao seu desempenho prévio (Farias et al., 2006).

O desempenho funcional dos pacientes com DA é a principal diferenciação para o quadro de CCL. Os adultos idosos com CCL, a partir dos critérios diagnósticos podem no máximo apresentar prejuízos sutis nas atividades diárias mais complexas, e/ou instrumentais (Albert et al., 2011; Marshall et al., 2011; Pereira, Yassuda, Oliveira, \& Forlenza, 2008; Teng, Becker, Woo, Cummings, \& Lu, 2010). Estas atividades funcionais complexas incluem a capacidade de preparar uma refeição, realizar trabalhos domésticos, cuidados com finanças e correspondência e administração da própria medicação. Por outro lado, as atividades de vida diárias básicas que compreendem o autocuidado e incluem a capacidade para realizar a higiene pessoal, o controle esfincteriano e a própria alimentação encontram-se preservadas (Chaves et al., 2011).

Assim, a avaliação funcional é de extrema importância para a diferenciação diagnóstica entre quadros demenciais iniciais e o CCL. Esta avaliação pode ser realizada através do relato de um informante por escalas e questionários, sendo considerada uma modalidade indireta de avaliação, ou pela observação e exame de desempenho funcional a partir da simulação de atividades de vida diária, isto é, de forma ecológica, considerada uma forma direta de avaliação (Lima-Silva et al., 2014; Pereira, Oliveira, et al., 2010). Tanto na prática clínica, quanto na pesquisa, a maioria dos instrumentos utilizados avaliam o desempenho funcional a partir dos relatos de um informante, o que pode subestimar ou superestimar o declínio funcional real do paciente. Isto porque são suscetíveis a vieses ocasionados por quadros de humor, personalidade, sentimentos negativos, cansaço físico ou pelo burnout do informante (Pereira, Oliveira, et al., 2010; Novelli, Nitrini, \& Caramelli, 2010). Além disso, diferente de uma avaliação realizada a partir do relato de um informante, a avaliação direta do desempenho em atividades diárias possibilita uma melhor acurácia na diferenciação entre adultos idosos saudáveis, com CCL e com DA (Goldberg et al., 2010).

Adicionalmente, a realização de uma avaliação funcional direta é de extrema importância considerando que cada vez mais os adultos idosos estão residindo sozinhos (Department of Health and Human Services [DHHS], 2002; Instituto Brasileiro de Geografia e Estatística [IBGE], 2008). Por isso, não possuem, na maioria das vezes, um familiar ou cuidador que esteja 
junto em sua rotina para observar os prejuízos funcionais. A realização deste tipo de avaliação funcional se faz necessária pensando-se em evitar situações de possíveis riscos a estes adultos idosos.

Diversas escalas e questionários foram desenvolvidos para a avaliação de atividades diárias básicas e complexas em indivíduos com demência (Johnson, Barion, Rademaker, Rehkemper, \& Weintraub, 2004). No Brasil alguns instrumentos para a avaliação funcional indireta estão validados e são recomendados para uso no diagnóstico de demência na DA (Chaves et al., 2011). São eles: Informant Questionnaire on Cognitive Decline in the Elderly (IQCODE; Bustamante et al., 2003; Jorm \& Jacomb, 1989; Sanchez, \& Lourenço, 2009); Disability Assessment for Dementia (DAD; Bahia et al., 2010; Gauthier et al., 1994); Activities of Daily Living Questionnaire (ADL-Q; Johnson et al., 2004; Medeiros \& Guerra, 2009); e Escala Bayer de Atividades da Vida Diária (B-ADL; Folquitto et al., 2007; Hindmarch, Lehfeld, de Jongh, \& Erzigkeit, 1998; Mapi Research Institute, 1999).

Dentre os instrumentos encontrados na literatura para a avaliação funcional o Activities of Daily Living Questionnaire (ADL-Q) é uma escala que avalia tanto as atividades básicas quanto as complexas (ou instrumentais) e é realizada com o familiar/ cuidador dos adultos idosos com suspeitas de um quadro demencial (Medeiros, \& Guerra, 2009). Ele avalia seis domínios que vão desde o autocuidado, interação social, organização, planejamento, até participação social. Esta é uma escala de observação do paciente a partir de um terceiro. Em síntese, ele depende do relato de um observador (Johnson et al., 2004). O único estudo encontrado avaliando pacientes com CCL e DA utilizando ADL-Q demonstrou uma alta confiabilidade teste-reteste e boa precisão para detectar um declínio leve, assim como os sintomas mais graves de progressão da doença (Johnson et al., 2004).

Para a avaliação funcional direta podem ser utilizados o instrumento Performance-Based Skills Assessment (Goldberg et al., 2010) e a Direct Assessment of Functional Status (DAFS) que foram utilizados com pacientes com queixas e suspeita de um quadro demencial (Loewenstein et al., 1989). A DAFS foi desenvolvida para avaliar uma variedade de capacidades funcionais, tanto básicas quanto complexas, necessárias para uma vida independente em pacientes adultos idosos com e sem comprometimento cognitivo. O principal objetivo da DAFS é realizar uma avaliação mais próxima do desempenho diário real dos adultos idosos (Loewenstein et al., 1989; Zanetti, Frisoni, Rozzini, Bianchetti, \& Trabucchi, 1998).

A DAFS avalia seis domínios orientação temporal, comunicação, habilidade para lidar com dinheiro e compras, higiene pessoal e capacidade para vestir-se e alimentar-se de forma independente (Pereira, Oliveira, et al., 2010). A validação da DAFS-R para a cultura brasileira foi realizada através de uma amostra de idosos saudáveis, pacientes com CCL e com DA. Neste estudo foi observada diferença significativa em pacientes com DA em relação aos adultos idosos saudáveis e CCL, mostrando menor desempenho na orientação, habilidade de comunicação e horário. As habilidades de vestir-se/higiene pessoal e alimentação apresentaram menores relações comparando com as outras subcategorias. Isto pode ter ocorrido pelo fato das tarefas envolvidas nestes domínios serem realizadas automaticamente, portanto, essas tarefas podem ter habilidades limitadas para diferenciar estes grupos de adultos idosos. A DAFS tem ótima precisão de discriminar idosos saudáveis de pacientes com DA (Loewenstein et al., 1989) e, ainda, diferencia idosos com CCL de idosos saudáveis (Pereira, Oliveira, et al., 2010).

Portanto, pode-se observar que a avaliação direta do desempenho funcional apresentou bom poder discriminativo entre adultos idosos saudáveis, pacientes com CCL e pacientes com DA (Pereira, Oliveira, et al., 2010). No entanto, no estudo de validação para o Brasil parece não ter havido a preocupação específica de somente incluir pacientes com demência em estágio leve. A inclusão de pacientes em estágios mais avançados da demência possibilita uma maior tendência à presença de discriminação entre os grupos. Além do mais, não foi encontrado estudo que tenha realizado avaliação da acurácia por 
domínios funcionais de avaliação direta. Portanto, o estudo também teve como objetivos avaliar a acurácia dos escores totais das avaliações direta e indireta entre adultos idosos com CCL, com DA e controles; e a acurácia dos domínios da avaliação direta entre Controles versus $\mathrm{CCL}$ e CCL versus DA, dos domínios que demonstrarem diferença no desempenho entre estes grupos.

\section{Método}

\section{Participantes}

A amostra do presente estudo foi composta por 90 adultos idosos, sendo divididos em três grupos: $n=27$ controles, $n=35$ pacientes com CCL e $n=28$ pacientes com DA leve. Os seguintes critérios de inclusão/ exclusão foram utilizados para os diferentes grupos: adultos idosos controles, adultos idosos com CCL e adultos idosos com DA. Critérios de inclusão para todos os grupos: indivíduos com 55 anos de idade ou mais e com pelo menos um ano completo de escolaridade formal. Os pacientes com CCL deveriam preencher os critérios diagnósticos para Comprometimento Cognitivo Leve (Winblad et al., 2004); os indivíduos com DA, deveriam preencher os critérios atuais da doença de Alzheimer provável ou possível (Frota et al., 2011), para os quadros de possível DA somente foram incluídos pacientes com histórico de doença vascular concomitante. Os pacientes com DA também deveriam estar apresentando estágio leve de gravidade avaliado pela Escala de Avaliação Clínica de Demência $(C D R)=1$ (Chaves et al., 2007; Hughes, Berg, Danziger, Coben, \& Martin, 1982) e Mini Exame do Estado Mental (MEEM)>14 pontos (Reisberg et al., 2011), ou MEEM $>14$ e ADL-Q $<66$ pontos (Medeiros, \& Guerra, 2009).

Para todos os grupos foram excluídos participantes com distúrbios sensoriais (auditivos e/ ou visuais) sem correção; história atual, ou prévia de abuso de álcool, de drogas ilícitas, quadros afásicos que impossibilitasse a avaliação. Para o grupo de controles e de CCL também foram excluídos participantes com histórico de quadros neurológicos ou psiquiátricos atuais; presença de sintomas depressivos atuais, maior ou igual a leve (GDS $\leq 6)$ para os controles e maior do que leve para CCL, avaliados pela Escala de Depressão Geriátrica (GDS-15) - (Almeida \& Almeida, 1999; Yesavage et al., 1983); desempenho cognitivo abaixo do esperado avaliado pelo Mini Exame do Estado Mental - MEEM (MEEM $\leq 22$, se escolaridade entre 1 e 5 anos, MEEM $\leq 23$, se escolaridade entre 6 e 11 anos, $M E E M \leq 24$, se escolaridade $\geq 12$ anos (Chaves, \& Izquierdo, 1992; Folstein, Folstein, \& McHugh, 1975; Kochhann, Varela, Lisboa, \& Chaves, 2010). Para o grupo de controles também foram excluídos participantes com nível intelectual igual ou inferior a limítrofe examinado pela escala WASI (Trentini, Yates, \& Heck, 2014; Wechsler, 1999).

A amostra inicial do estudo foi de 123 indivíduos, destes, 18 pacientes foram excluídos por apresentarem quadro demencial de moderado a grave, cinco participantes por apresentarem prejuízo cognitivo devido à outra doença neurológica que não DA (ex.: doença de Parkinson, TCE, demência alcoólica), nove indivíduos por apresentarem transtorno psiquiátrico atual, e um sujeito por apresentar QI limítrofe.

\section{Aspectos Éticos e Procedimentos de Coleta}

O presente estudo foi realizado como parte de um projeto guarda-chuva aprovado pelo Comitê de Ética em Pesquisa da Pontifícia Universidade Católica do Rio Grande do Sul (PUCRS), número 657.955/14 e pelo Comitê de Ética do Hospital de Clínicas de Porto Alegre (HCPA), número 14-0368. Os pacientes foram recrutados do Ambulatório de Demências de Hospital das Clínicas de Porto Alegre e por divulgação em televisão, rádio e internet. Todos os participantes assinaram e receberam uma via do Termo de Comprometimento Livre e Esclarecido. Todos os participantes adultos idosos e seus familiares/ cuidadores foram avaliados individualmente em salas silenciosas e livre de estímulos externos. A avaliação teve duração de duas sessões de duas horas, aproximadamente, seguindo a mesma ordem dos instrumentos para todos os participantes. A escala CDR não foi aplicada durante esse estudo, pois ela é utilizada em sua rotina nos atendimentos aos pacientes do Ambulatório de 
Demência do HCPA, de onde eles foram provenientes. Os prontuários dos pacientes atendidos nesse ambulatório foram avaliados e somente pacientes com diagnóstico de demência da DA com estágio classificado como leve $(\mathrm{CDR}=1)$ foram convidados a participar do presente estudo.

\section{Instrumentos}

Os instrumentos foram divididos, para melhor compreensão, em instrumentos de caracterização da amostra e instrumentos de avaliação funcional.

Instrumentos de Caracterização da Amostra. Questionário sociocultural e de aspectos de saúde para adultos idosos é um questionário semiestruturado que engloba questões sobre sexo; idade; escolaridade; verificação da frequência dos hábitos de leitura e escrita através das pontuações: diária (4 pontos), semanal ( 3 pontos), mensal ( 2 pontos), raramente ( 1 ponto) e nunca (zero pontos), apresentando pontuação total de 28 pontos; uso de medicamentos; descrição do início e progressão dos sintomas de esquecimento; ou prejuízo funcional. Avalia também o nível socioeconômico de acordo com as regras da Associação Brasileira de Empresas de Pesquisa (ABEP, 2014), considerando os estratos A (45 a 100 pontos), B1 (38 a 44 pontos), B2 (29 a 37 pontos), C1 (23 a 28 pontos), C2 (17 a 22 pontos) e D-E ( 0 a 16 pontos) entre outras variáveis clínicas e sociodemográficas.

Os demais instrumentos utilizados constam na descrição da amostra e foram utilizados para a avaliação dos critérios de inclusão e exclusão dos participantes. Adicionalmente, todos os participantes realizaram avaliação neuropsicológica ampla de memória episódica verbal e visual, memória semântica, memória prospectiva, memória de trabalho, avaliação da capacidade de iniciação e inibição verbal e visuomotora, e de habilidades linguísticas de fluência verbal, compreensão e nomeação oral (Fransen et al., no prelo).

\section{Instrumentos de Avaliação Funcional}

Activities of Daily Living Questionnaire (ADL-Q; Johnson et al., 2004; Medeiros \& Guerra, 2009) é um instrumento que avalia o funcionamento na rotina do paciente através das informações fornecidas por familiar ou cuidador. Permite avaliar atividades de vida diárias básicas e instrumentais (domínios de autocuidado, interação, atividade intelectiva, organização/ planejamento, participação social e alimentação). A pontuação total varia de 0 a 100. Sendo classificada de 0 a 33 sem prejuízo a prejuízos leves; de 34 a 66 prejuízos moderados; e acima de 66 prejuízos graves. A versão adaptada para o Brasil apresentou boa confiabilidade através do alpha de Cronbach de 0,759 (Medeiros, \& Guerra, 2009).

Direct Assessment of Functional Status - Revised (DAFS-R; Loewenstein et al., 1989; Pereira, Oliveira, et al., 2010) é um instrumento baseado em simulações das atividades de vida diária básicas e instrumentais do adulto idoso. Em que o participante deve realizar algumas atividades semelhantes a sua rotina. A DAFS-R é uma versão abreviada que avalia sete domínios diferentes de habilidades funcionais. A pontuação total varia de 0 a 105, sendo quando maior a pontuação, melhor o desempenho. Os domínios avaliados são orientação temporal (16 pontos); habilidades necessárias para a comunicação (14 pontos); lidar com as finanças (32 pontos); habilidades de compra (20 pontos); habilidades necessárias para se arrumar/vestir (13 pontos) e comer (10 pontos). A versão adaptada para o Brasil apresentou boa sensibilidade $=0.995$ e especificidade $=0,995$ para identificar CCL e DA (Pereira, Yassuda, et al., 2010).

\section{Análise de Dados}

Para tabulação e análise de dados utilizouse o Statistical Package for the Social Sciences (SPSS) versão 17.0. As variáveis analisadas não apresentaram uma distribuição normal. Dessa forma, foram realizados os testes não paramétricos Teste Kruskal Wallis e o Qui-quadrado para a caracterização da amostra. Após foi realizado uma MANCOVA controlando-se anos de idade, e de escolaridade, a frequência de hábitos de leitura e escrita e o escore do nível socioeconômico para verificar o desempenho funcional direto dos participantes nos grupos (Controle versus CCL e 
CCL versus DA), incluindo o escore geral e dos domínios. Foi realizada uma análise de correlação de Spearman entre a ADL-Q e a DAFS-R a fim de verificar a possível associação entre elas. Por fim, para verificar a acurácia diagnóstica das escalas funcionais no ADL-Q e na DAFS-R entre os grupos Controles versus CCL e CCL versus DA foram realizadas curvas ROC (receiver operating characteristic). Para todas as análises consideraram-se significativas às diferenças com nível de significância $p \leq 0,05$.

\section{Resultados}

Os dados sociodemográficos e clínicos da amostra podem ser observados na Tabela 1. Entre as variáveis avaliadas não houve diferença na distribuição do sexo. Nas outras variáveis, pode-se observar que os pacientes com DA eram mais velhos, com menos escolaridade, tinham menor escore de classe econômica, menos hábitos de leitura e escrita, e maior prejuízo cognitivo.

Tabela 1

Dados Sociodemográficos e Clínicos da Amostra

\begin{tabular}{lccccc}
\hline \multicolumn{1}{c}{ Variáveis } & $\begin{array}{c}\text { Controle } \\
(n=27)\end{array}$ & $\begin{array}{c}\mathrm{CCL} \\
(n=35)\end{array}$ & $\begin{array}{c}\mathrm{DA} \\
(n=28)\end{array}$ & $\begin{array}{c}p \\
\text { Análise } \\
\text { Post-Hoc }\end{array}$ \\
\hline Idade $(m \pm d p)$ & $68,07 \pm 5,57$ & $69,34 \pm 7,04$ & $75,07 \pm 6,65$ & $\mathbf{0 , 0 0 1}$ & $\mathrm{C}, \mathrm{CCL}<\mathrm{DA}$ \\
Escolaridade $(m \pm d p)$ & $14,19 \pm 5,57$ & $10,26 \pm 4,60$ & $6,71 \pm 5,16$ & $<\mathbf{0 , 0 0 1}$ & $\mathrm{C}>\mathrm{CCL}>\mathrm{DA}$ \\
Escore total classe econômica $(m \pm d p)$ & $36,08 \pm 12,17$ & $32,34 \pm 10,14$ & $27,93 \pm 6,76$ & $\mathbf{0 , 0 3 8}$ & $\mathrm{C}>\mathrm{DA}$ \\
Escore Total Hábitos de Leitura & $16,22 \pm 4,91$ & $11,97 \pm 6,35$ & $5,79 \pm 4,22$ & $<\mathbf{0 , 0 0 1}$ & $\mathrm{C}>\mathrm{CCL}>\mathrm{DA}$ \\
e Escrita $(m \pm d p)$ & $28,41 \pm 2,39$ & $26,11 \pm 3,47$ & $21,04 \pm 4,01$ & $<\mathbf{0 , 0 0 1}$ & $\mathrm{C}>\mathrm{CCL}>\mathrm{DA}$ \\
MEEM $(m \pm d p)$ & $1,59 \pm 1,86$ & $2,06 \pm 2,26$ & $4,96 \pm 3,46$ & $<\mathbf{0 , 0 0 1}$ & $\mathrm{C}, \mathrm{CCL}<\mathrm{DA}$ \\
GDS-15 $(m \pm d p)$ & $20(74,1)$ & $25(71,4)$ & $22(78,6)$ & 0,811 & - \\
Sexo feminino - $n(\%)$ & & &
\end{tabular}

Nota. $p \leq 0,05$ é considerado significativo. MEEM= Mini Exame do Estado Mental; GDS-15= Escala de Depressão Geriátrica; $\mathrm{C}=$ Controle; $\mathrm{CCL}=$ Comprometimento Cognitivo Leve; $\mathrm{DA}=$ demência da doença de Alzheimer. Em negrito está realçado os valores com diferença significativa.

Tabela 2

Comparação do Desempenho Funcional Direto entre os Grupos

\begin{tabular}{lcccccc}
\hline & \multicolumn{3}{c}{ Controles X CCL } & & \multicolumn{2}{c}{ CCL X DA } \\
\cline { 2 - 7 } \multicolumn{1}{c}{ Variáveis } & $F$ & $p$ & $n^{2}$ partial & $F$ & $p$ & $n^{2}$ partial \\
\hline Domínio orientação & 1,045 & 0,311 & 0,019 & 29,748 & $<\mathbf{0 , 0 0 1}$ & 0,347 \\
Domínio comunicação & 0,041 & 0,840 & 0,001 & 4,400 & $\mathbf{0 , 0 4 0}$ & 0,073 \\
Domínio habilidades com dinheiro & 6,076 & $\mathbf{0 , 0 1 7}$ & 0,099 & 15,774 & $<\mathbf{0 , 0 0 1}$ & 0,220 \\
Domínio habilidades com compras & 0.957 & 0,332 & 0,17 & 13,906 & $<\mathbf{0 , 0 0 1}$ & 0,199 \\
Domínio vestir e higiene & 0,037 & 0,848 & 0,001 & 8,088 & $\mathbf{0 , 0 0 6}$ & 0,126 \\
Domínio alimentar-se & - & - & - & 2,007 & 0,162 & 0,035 \\
DAFS-R escore geral & 4,747 & $\mathbf{0 , 0 3 4}$ & 0,079 & 27,910 & $<\mathbf{0 , 0 0 1}$ & 0,333 \\
\hline
\end{tabular}

Nota. $p \leq 0,05$ é considerado significativo. DAFS-R= Direct Assessment of Functional Status - Revised; CCL= Comprometimento cognitivo leve; $\mathrm{DA}=$ demência da doença de Alzheimer. Em negrito está realçado os valores com diferença significativa. 
Nas análises de MANCOVA para verificar o desempenho funcional direto dos participantes nos grupos (Controle versus CCL e CCL versus DA), incluindo o escore geral e dos domínios, pode-se observar que somente o escore geral e o domínio de habilidades com dinheiro diferenciou controles de CCL, sendo que o grupo CCL apresentou escores mais baixos do que o grupo controle. Já na comparação entre CCL e DA tanto o escore geral e os domínios diferenciaram estes grupos, sendo que o grupo DA apresentou escores mais baixos do que o grupo CCL, com exceção do domínio alimentar-se no qual não houve diferenças entre os grupos (Tabela 2).

A análise de correlação de Spearman demonstrou que o escore total da DAFS-R e o esco- re total da ADL-Q apresentaram uma associação forte e negativa ( $\mathrm{rho}=-0,743, p<0,001$ ). Assim a partir disso, na Tabela 3, constam os melhores pontos de corte observados, mantendo-se o melhor balanceamento entre sensibilidade e especificidade para as escalas de avaliação funcional indireta e direta. Pode-se observar que ambas as escalas possuem maior acurácia entre os grupos CCL e DA. Contudo, a DAFS-R apresentou melhor acurácia para diferenciar controles de CCL do que a ADL-Q. Para a escala DAFS-R também foram realizadas análises de acurácia para os domínios que apresentaram diferença de desempenho entre Controles versus CCL e CCL versus DA na análise de MANCOVA.

Tabela 3

Pontos de Corte das Escalas DAFS - R e ADL - Q

\begin{tabular}{|c|c|c|c|c|c|}
\hline Grupos & Domínios & Pontos de corte & Área sobre a curva & Sensibilidade & Especificidade \\
\hline \multicolumn{6}{|c|}{ DAFS - R } \\
\hline \multirow{4}{*}{$\begin{array}{c}\text { Controles } \\
\text { versus } \\
\text { CCL }\end{array}$} & Escore geral & 91 & 0,726 & 68 & 63 \\
\hline & $\begin{array}{l}\text { Habilidades } \\
\text { com dinheiro }\end{array}$ & 26 & 0,744 & 68 & 66 \\
\hline & Escore geral & 70 & 0,905 & 89 & 83 \\
\hline & Orientação & 13 & 0,916 & 89 & 77 \\
\hline \multirow{5}{*}{$\begin{array}{c}\text { CCL } \\
\text { versus } \\
\text { DA }\end{array}$} & Comunicação & 10 & 0,792 & 78 & 71 \\
\hline & $\begin{array}{l}\text { Habilidades } \\
\text { com dinheiro }\end{array}$ & 19 & 0,871 & 89 & 77 \\
\hline & $\begin{array}{l}\text { Habilidades } \\
\text { com compras }\end{array}$ & 11 & 0,848 & 89 & 77 \\
\hline & Vestir e Higiene & 12 & 0,758 & 71 & 68 \\
\hline & \multicolumn{4}{|c|}{$\mathrm{ADL}-\mathrm{Q}$} & \\
\hline $\begin{array}{c}\text { Controles } \\
\text { versus } \\
\text { CCL }\end{array}$ & Escore geral & 1 & 0,653 & 66 & 59 \\
\hline $\begin{array}{c}\text { CCL } \\
\text { versus } \\
\text { DA }\end{array}$ & Escore geral & 21 & 0,977 & 93 & 91 \\
\hline
\end{tabular}

Nota. $p \leq 0,05$ é considerado significativo. ADL-Q = Activities of Daily Living Questionnaire DAFS-R= Direct Assessment of Functional Status - Revised; $\mathrm{CCL}=$ Comprometimento cognitivo leve; $\mathrm{DA}=$ demência da doença de Alzheimer. 


\section{Discussão}

O estudo teve como objetivo comparar a acurácia dos escores totais da avaliação direta pela DAFS e da avaliação indireta pela escala ADL-Q entre adultos idosos com CCL, com DA e controles, e averiguar a acurácia dos domínios da avaliação direta entre Controles versus CCL e CCL versus DA. A comparação do desempenho da avaliação direta representada pelo instrumento DAFS, controlando as variáveis sociodemográficas, demonstrou que o escore total é capaz de diferenciar controles de CCL e CCL de DA. No entanto, apesar de quase todos os domínios se diferenciarem entre os grupos de CCL e de DA, somente pode ser observado que as habilidades com dinheiro diferenciaram os grupos Controles de CCL. Este resultado indica que as habilidades de lidar com dinheiro podem ser o primeiro indício de declínio na funcionalidade muito sutil que já está presente nos quadros de CCL. Isso sugere que indivíduos com CCL possuem risco aumentado para desenvolver demência (Boyle et al., 2006), justamente por já começar a apresentar pequenos declínios em habilidades instrumentais que antes não tinham.

A avaliação pela DAFS além de ter diferenciado controles de CCL, replicando estudos prévios (Pereira, Yassuda, et al., 2010; Puente, Terry, Faraco, Brown, \& Miller, 2014), também apresentou ótima acurácia para diferenciar CCL de DA. Ainda, pode ser observado que a avaliação direta da funcionalidade apresenta melhor acurácia do que uma avaliação indireta. Provavelmente por capturar mais informações sobre a gravidade global da demência (Farias, Harrell, Neumann, \& Houtz, 2003).

Em relação à avaliação dos domínios da DAFS, os resultados corroboram em parte com os de outros estudos que diferenciaram indivíduos controles do grupo com CCL no domínio de habilidades com dinheiro (Pereira, Oliveira, et al., 2010; Razani et al., 2011); porém, no estudo de Pereira, Oliveira, et al. (2010) encontrou-se diferença em habilidades de compras. Contudo, vale ressaltar que este estudo utilizou como critério de exclusão escores inferiores a 10 pontos no MEEM e considerou a inclusão de DA em estágios inicial, leve ou moderada, sendo que no presente estudo os critérios de exclusão foram MEEM acima de 14 pontos e critérios de inclusão apenas DA em estágio leve. Assim, o objetivo do presente estudo foi viabilizar a diferenciação entre controles e CCL e entre CCL e DA, a fim de avaliar com maior precisão o declínio funcional presente nos quadros de neurodegeneração cognitiva.

Estudos evidenciaram que adultos idosos com DA mostraram maior prejuízos nos domínios de habilidades financeiras, de compras, orientação temporal e habilidade de comunicação em relação aos idosos saudáveis e CCL (Goldberg et al., 2010; Pereira, Oliveira, et al., 2010). Os achados demonstraram resultados similares, pela avaliação direta, adultos idosos com DA apresentaram pior desempenho em relação aos pacientes com CCL no escore total e em todos os domínios, com exceção do domínio alimentar-se. Já em relação à diferenciação entre CCL e controles somente a DAFS demonstrou ser capaz de diferenciar estes adultos idosos. Assim, este tipo de avaliação funcional direta demonstra ser de extrema importância para a diferenciação destas patologias.

A vantagem percebida em informações baseadas diretamente com o paciente é que estão livres das possíveis distorções da avaliação do cuidador e das possíveis sub e superestimação de sua funcionalidade (Goldberg et al., 2010; Ready, Ott, \& Grace, 2004), assim como, não estão suscetíveis a vieses ocasionados por quadros de humor, personalidade ou pelo burnout dos informantes (Pereira, Oliveira, et al., 2010). Além disso, adultos idosos que residem sozinhos podem não ter um informante confiável por este não ter uma frequência adequada de contato que permita um relato acurado do desempenho funcional, assim, não possuem um familiar que esteja junto em sua rotina para observar os prejuízos funcionais. Um estudo demonstrou que os cuidadores que passavam menos de 20 horas por semana com os adultos idosos estavam inclinados a fazer pelo menos um erro de julgamento (Loewenstein et al., 2001) o que enfatiza a ne- 
cessidade da realização de avaliação funcional direta principalmente em adultos idosos que residem sozinhos.

De acordo com Department of Health and Human Services (DHHS, 2002), os adultos idosos têm o desejo de manter a independência e residir sozinhos, e com o aumento da idade o percentual de idosos que vivem sozinhos aumenta proporcionalmente (DHHS, 2002; IBGE, 2008). Assim, devido ao aumento progressivo de idosos que moram sozinhos há a necessidade da realização de uma avaliação funcional direta, possibilitando um melhor acompanhamento e realização de diagnóstico precoce a fim de proporcionar tratamento adequado para os quadros demenciais.

Uma limitação encontrada foi à inclusão da escala ADL-Q no objetivo principal do estudo. Isto porque a escala pertencia aos instrumentos que avaliavam os critérios de inclusão para a amostra (auxiliando na diferenciação entre CCL e DA), mas ao mesmo tempo foi realizada como critério de acurácia de avaliação indireta, ou seja, medida de desfecho principal: funcionalidade. Contudo, isto foi realizado por não ter sido encontrado nenhum artigo com pontos de corte da ADL-Q. O estudo de validação da escala ADL-Q demonstrou que ela é sensível à detecção de declínio leve, bem como de prejuízos mais graves de progressão da doença (Johnson et al., 2004). Contudo, este estudo não incluiu grupo controle e CCL em sua amostra (Johnson et al., 2004). No presente estudo, embora a ADL-Q tenha demonstrado boa acurácia para discriminar quadros de CCL e DA, não apresentou poder discriminativo para a diferenciação entre adultos idosos controles e com CCL. É importante ressaltar que as pontuações da ADL-Q e da DAFS são opostas, ou seja, quanto melhor o desempenho na ADL-Q será próximo ou igual ao escore zero e na DAFS é o contrário, o escore zero indica o pior desempenho. Este aspecto implica em se ter pouca ou quase nenhuma variabilidade na pontuação da escala ADL-Q entre controles e CCL. De maneira geral, esta é uma característica das escalas que avaliam a funcionalidade através do relato de um informante.
Assim, pode-se concluir que a avaliação do desempenho funcional realizada diretamente com os pacientes apresenta melhor acurácia diagnóstica entre adultos idosos controles e CCL e entre idosos com CCL e DA. Assim, este tipo de instrumento demonstra ser imprescindível na avaliação de idosos que não possuem informantes ou que os mesmos não possuam relato confiável. Salienta-se a importância de seu uso também em contexto de saúde pública, com o objetivo de aumentar a precisão diagnóstica. Contudo, recomenda-se a realização de estudos que investiguem os níveis funcionais para cada subtipo de CCL. Adicionalmente, sugere-se a realização de estudos longitudinais para avaliar o continuum funcional dos subgrupos que foram avaliados no presente estudo.

\section{Contribuições dos Autores}

Contribuição substancial no conceito e desenho do estudo: Natalia Lemos Fransen, Rochele Paz Fonseca, Renata Kochhann.

Contribuição na coleta de dados: Natalia Lemos Fransen, Maila Holz, Andressa Pereira.

Contribuição na análise e interpretação de dados: Natalia Lemos Fransen, Maila Holz, Andressa Pereira, Renata Kochhann.

Contribuição para a preparação do manuscrito: Natalia Lemos Fransen, Maila Holz, Andressa Pereira, Renata Kochhann.

Contribuição à revisão crítica, agregando conteúdo intelectual: Rochele Paz Fonseca.

\section{Conflitos de interesse}

Os autores declaram não ter conflito de interesse relacionado à publicação deste manuscrito.

\section{Referências}

Albert, M. S., Dekosky, S. T., Dickson, D., Dubois, B., Feldman, H. H., Fox, N. C., ...Phelps, C. H. (2011). The diagnosis of mild cognitive impairment due to Alzheimer's disease: Recommendations from the National Institute on Aging-Alzheimer's Association workgroups on diagnostic guidelines for Alzheimer's dis- 
ease. Alzheimer's \& Dementia, 7, 270-279. doi: 10.1016/j.jalz.2011.03.008

Almeida, O. P., \& Almeida, S. A. (1999). Confiabilidade da versão brasileira da Escala de Depressão em Geriatria (GDS) versão reduzida. Arquivos de Neuro-Psiquiatria, 57(2B), 421-426. doi: 10.1590/S0004-282X1999000300013

American Psychiatric Association. (2014). Manual diagnóstico e Estatístico de Transtornos Mentais: DSM-5. Porto Alegre, RS: Artmed

Associação Brasileira de Empresas de Pesquisa. (2014). Critério de Classificação Econômica Brasil. Recuperado em http://www.abep.org

Bahia, V., S., Carthery-Goulart, M., T., Novelli, M., M., Kato-Narita, E. M., Areza-Fegyveres, R., Caramelli, P., \& Nitrini, R. (2010). Functional disability in Alzheimer disease: a validation study of the Brazilian version of Disability Assessment for Dementia (DAD-Br). Alzheimer Disease and Associated Disorder, 24, 291-295. doi: 10.1097/WAD.0b013e3181 cfc878

Boyle, P. A., Wilson, R. S., Aggarwal, N. T., Tang, Y., \& Bennett, D. A. (2006). Mild cognitive impairment Risk of Alzheimer disease and rate of cognitive decline. Neurology, 67(3), 441-445. doi: 10.1212/01.wnl.0000228244.10416.20

Bustamante, S., E., Z., Bottino, C., M., C., Lopes, M., A., Azevedo, D., Hototian, S. R., Litvoc, J., \& Jacob, W., Filho. (2003). Instrumentos combinados na avaliação de demência de idosos. Arquivos de Neuropsiquiatria, 61, 601606. doi: http://dx.doi.org/10.1590/S0004$-282 \times 2003000400014$

Chaves, M. L. F., \& Izquierdo, I. (1992). Differential diagnosis between dementia and depression: A study of efficiency increment. Acta Neurologica Scandinavica, 85(6), 378-382. doi: 10.1111/ j.1600-0404.1992.tb06032.x

Chaves, M. L. F., Camozzato, A. L., Godinho, C., Kochhann, R., Schuh, A., De Almeida, V. L., \& Kaye, J. (2007). Validity of the clinical dementia rating scale for the detection and staging of dementia in Brazilian patients. Alzheimer Disease \& Associated Disorders, 21(3), 210-217. doi: 10.1097/WAD.0b013e31811ff2b4

Chaves, M. L., Godinho, C. C., Porto, C. S., Mansur, L., Carthery-Goulart, M. T., Yassuda, M. S., \& Beato, R. (2011). Doença de Alzheimer: Avaliação cognitiva, comportamental e funcional. Dementia \& Neuropsychologia, 5(Suppl 1).
Department of Health and Human Services. (2002). Annual Report. Retrieved from https://www. acl.gov/sites/default/files/about-acl/2017-04/ AOA_2002_AnnualReport.pdf

Farias, S. T., Harrell, E., Neumann, C., \& Houtz, A. (2003). The relationship between neuropsychological performance and daily functioning in individuals with Alzheimer's disease: Ecological validity of neuropsychological tests. Archives of Clinical Neuropsychology, 18(6), 655-672. doi: 10.1016/S0887-6177(02)00159-2

Farias, S. T., Mungas, D., Reed, B. R., Harvey, D., Cahn-Weiner, D., \& DeCarli, C. (2006). MCI is associated with deficits in everyday functioning. Alzheimer Disease and Associated Disorders, 20(4), 217. doi: 10.1097/01. wad.0000213849.51495.d9

Folquitto, J., C., Bustamente, S., E., Z., Barros, S., B., Azevedo, D., Lopes, M. A., Hototian, S. R., ...Bottino, C. M. C. (2007). The Bayer- Activities of daily living scale (B-ADL) in the differentiation between mild to moderate dementia and normal aging. Revista Brasileira de Psiquiatria, 29, 350-353. doi: http://dx.doi.org/10.1590/ S1516-44462006005000037

Folstein, M. F., Folstein, S. E., \& McHugh, P. R. (1975). "Mini-mental state": A practical method for grading the cognitive state of patients for the clinician. Journal of Psychiatric Research, 12(3), 189-198. doi: 10.1016/0022-3956(75)90026-6

Fransen, N., Holz, M., Tarrasconi, M., Candemil, C., Fonseca, R. P., \& Kochhann, R. (no prelo). Fatores cognitivos como preditores da funcionalidade no contínuo de comprometimento cognitivo no envelhecimento: Memória de trabalho e memória episódica verbal.

Frota, N. A. F., Nitrini, R., Damasceno, B.P., Forlenza, O., Dias-Tosta, E., da Silva, A. B., ...Magaldi, R. M. (2011). Critérios para o diagnóstico de doença de Alzheimer. Dementia \& Neuropsychologia, 5(Suppl. 1), 5-10.

Gauthier, L., Gélinas, I., McIntyre, M., Gauthier, S., Laberge, H., \& Dauphinee, S., W. (1994). Disability Assessment for Dementia (DAD) user's guide.

Goldberg, T. E., Koppel, J., Keehlisen, L., Christen, E., Dreses-Werringloer, U., Conejero-Goldberg, C., \& Davies, P. (2010). Performance-based measures of everyday function in mild cognitive impairment. American Journal of Psy- 
chiatry, 167(7), 845-853. doi: 10.1176/appi. ajp.2010.09050692

Hindmarch, I., Lehfeld, H., de Jongh, P., Erzigkeit, H. (1998). The Bayer Activities of Daily Living Scale (B-ADL). Dementia and Geriatric Cognitive Disorders, 9(2), 20-26.

Hughes, C. P., Berg, L., Danziger, W. L., Coben, L. A., \& Martin, R. (1982). A new clinical scale for the staging of dementia. The British Journal of Psychiatry, 140(6), 566-572. doi: 10.1192/ bjp.140.6.566

Instituto Brasileiro de Geografia e Estatística. (2008). Síntese de Indicadores Sociais: Uma análise das condições de vida da população brasileira. Estudos e Pesquisas: Informação Demográfica e Socioeconômica, 23.

Johnson, N., Barion, A., Rademaker, A., Rehkemper, G., \& Weintraub, S. (2004). The Activities of Daily Living Questionnaire: A validation study in patients with dementia. Alzheimer Disease \& Associated Disorders, 18(4), 223-230.

Jorm, A. F., \& Jacomb, P. A. (1989). The Informant Questionnaire on Cognitive Decline in the Elderly (IQCODE): Socio-demographic correlates, reliability, validity and some norms. Psychological Medicine, 19, 1015-1022.

Kochhann, R., Varela, J. S., Lisboa, C. S., \& Chaves, M. L. F. (2010). The Mini Mental State Examination: Review of cutoff points adjusted for schooling in a large Southern Brazilian sample. Dementia \& Neuropsychologia, 35-41.

Lima-Silva, T., B., Bahia, V., S., Carvalho, V., A., Guimarães, H., C., Caramelli, P., Balthazar, M., L., F., ...Yassuda, M. S. (2014). Direct and Indirect Assessments of Activities of Daily Living in Behavioral Variant Frontotemporal Dementia and Alzheimer Disease. Journal of Geriatric Psychiatry and Neurology, 28, 1-8. doi: 10.1177/0891988714541874

Loewenstein, D. A., Amigo, E., Duara, R., Guterman, A., Hurwitz, D., Berkowitz, N., Wilkie, F., .. Eisdorfer, C. (1989). A new scale for the assessment of functional status in Alzheimer's disease and related disorders. Journal of Gerontology, 44(4), 114-121. doi: https://doi.org/10.1093/ geronj/44.4.P114

Loewenstein, D. A., Argüelles, S., Bravo, M., Freeman, R. Q., Argüelles, T., Acevedo, A., \& Eisdorfer, C. (2001). Caregivers' Judgments of the Functional Abilities of the Alzheimer's
Disease Patient a Comparison of Proxy Reports and Objective Measures. The Journals of Gerontology Series B: Psychological Sciences and Social Sciences, 56(2), 78-84.

Mapi Research Institute. (1999). Cultural Adaptation of the Bayer Activities of Daily Living Scale (B$A D L)$ into Brazilian Portuguese (Report). Lyon, France: Author.

Marshall, G. A., Rentz, D. M., Frey, M. T., Locascio, J. J., Johnson, K. A., Sperling, R. A., \& Alzheimer's Disease Neuroimaging Initiative. (2011). Executive function and instrumental activities of daily living in mild cognitive impairment and Alzheimer's disease. Alzheimer's \& Dementia, 7(3), 300-308. doi: 10.1016/j.jalz.2010.04.005

McKhann, G. M., Knopman, D. S., Chertkow, H., Hyman, B. T., Jack, C. R., Kawas, C. H., Klunk, W. E., ...Mohs, R. C. (2011). The diagnosis of dementia due to Alzheimer's disease: Recommendations from the National Institute on Aging-Alzheimer's Association workgroups on diagnostic guidelines for Alzheimer's disease. Alzheimer's \& Dementia, 7(3), 263-269. doi: 10.1016/j.jalz.2011.03.005

Medeiros, M. E. D., \& Guerra, R. O. (2009). Tradução, adaptação cultural e análise das propriedades psicométricas do Activities of Daily Living Questionnaire (ADLQ) para avaliação funcional de pacientes com a doença de Alzheimer. Revista Brasileira Fisioterapia, 13(3), 257-66.

Novelli, M. M. P. C., Nitrini, R., \& Caramelli, P. (2010). Cuidadores de idosos com demência: Perfil sociodemográfico e impacto diário. Revista de Terapia Ocupacional da Universidade de São Paulo, 21(2), 139-147.

Pereira, F. S., Oliveira, A. M., Diniz, B. S., Forlenza, O. V., \& Yassuda, M. S. (2010). Cross-cultural adaptation, reliability and validity of the DAFS$\mathrm{R}$ in a sample of Brazilian older adults. Archives of clinical neuropsychology, 25(4), 335-343. doi: 10.1093/arclin/acq029

Pereira, F. S., Yassuda, M. S., Oliveira, A. M., \& Forlenza, O. V. (2008). Executive dysfunction correlates with impaired functional status in older adults with varying degrees of cognitive impairment. International Psychogeriatrics, 20(6), 1104. doi: 10.1017/S1041610208007631

Pereira, F. S., Yassuda, M. S., Oliveira, A., Diniz, B. S., Radanovic, M., Talib, L. L., Gattaz, W. F., \& Forlenza, O. V. (2010). Profiles of func- 
tional deficits in mild cognitive impairment and dementia: benefits from objective measurement. Journal of the International Neuropsychological Society, 16(02), 297-305. doi: 10.1017/ S1355617709991330

Petersen, R., C., Roberts, R., O., Knopman, D., Boeve, B. F., Geda, Y. E., Ivnik, R. J., ...Jack, C. R., Jr. (2009). Mild cognitive impairment: Ten years later. Archives of Neurology, 66, 14471455. doi: 10.1001/archneurol.2009.266

Puente, A. N., Terry, D. P., Faraco, C. C., Brown, C. L., \& Miller, L. S. (2014). Functional impairment in mild cognitive impairment evidenced using performance-based measurement. Journal of Geriatric Psychiatry and Neurology, 27(4), 253-258. doi: 10.1177/0891988714532016

Razani, J., Bayan, S., Funes, C., Mahmoud, N., Torrence, N., Wong, J., ...Josephson, K. (2011). Patterns of deficits in daily functioning and cognitive performance of patients with Alzheimer disease. Journal of Geriatric Psychiatry and Neurology, 24(1), 23-32. doi: 10.1177/0891988710390812

Ready, R. E., Ott, B. R., \& Grace, J. (2004). Patient versus informant perspectives of quality of life in mild cognitive impairment and Alzheimer's disease. International Journal of Geriatric Psychiatry, 19(3), 256-265. doi: 10.1002/gps.1075

Reisberg, B., Jamil, I. A., Khan, S., Monteiro, I., Torossiani, C., Ferris, S., ...Wegiels, J. (2011). Staging Dementia. In J. R. M. Copeland, M. T. Abou-Saleh, \& D. G. Blazer (Eds.), Principles and Practice of Geriatric Psychiatry ( $3^{\text {rd }}$ Ed.). Hoboken, NJ: John Wiley \& Sons.

Sanchez, M., A., \& Lourenço, R., A. (2009). Informant Questionnaire on Cognitive Decline in the Elderly (IQCODE): Cross-cultural adaptation for use in Brazil. Cadernos de Saúde Pública, $25,1455-1465$.
Teng, E., Becker, B. W., Woo, E., Cummings, J. L., \& Lu, P. H. (2010). Subtle deficits in instrumental activities of daily living in subtypes of mild cognitive impairment. Dementia and Geriatric Cognitive Disorders, 30(3), 189-197. doi: $10.1159 / 000313540$

Trentini, C. M., Yates, D. B., \& Heck, V. S. (2014). WASI - Escala Wechsler Abreviada de Inteligência. São Paulo, SP: Pearson.

Wechsler, D. (1999). Wechsler Abbreviated Scale of Intelligence. San Antonio, TX: Pearson.

Winblad, B., Palmer, K., Kivipelto, M., Jelic, V., Fratiglioni, L., Wahlund, L.-O., ...Petersen, R. C. (2004). Mild cognitive impairment - Beyond controversies, towards a consensus: report of the International Working Group on Mild Cognitive Impairment. Journal of Internal Medicine, 256(3), 240-246.

Yesavage, J. A., Brink, T. L., Rose, T. L., Lum, O., Huang, V., Adey, M., \& Leirer, V. O. (1983). Development and validation of a geriatric depression screening scale: A preliminary report. Journal of Psychiatric Research, 17(1), $37-49$.

Zanetti, O., Frisoni, G. B., Rozzini, L., Bianchetti, A., \& Trabucchi, M. (1998). Validity of direct assessment of functional status as a tool for measuring Alzheimer's disease severity. Age and Ageing, 27(5), 615-622.
Recebido: 19/05/2017

$1^{a}$ revisão: 03/11/2017

Aceite final: 16/11/2017

(cc)BY (c) O(s) autor(es), 2018. Acesso aberto. Este artigo está distribuído nos termos da Licença Internacional Creative Commons Atribuição 4.0 (http://creativecommons.org/licenses/by/4.0/), que permite o uso, distribuição e reprodução sem restrições em qualquer meio, desde que você dê crédito apropriado ao(s) autor(es) original(ais) e à fonte, fornecer um link para a licença Creative Commons e indicar se as alterações foram feitas. 